\title{
Was heißt, sich im Wollen orientieren?
}

\author{
Gottfried Seebaß
}

\section{Fragestellung}

Der Titel meines Aufsatzes ist ein Plagiat. Er variiert den Titel eines Aufsatzes von Kant, in dem dieser die These vertritt, daß unser Denken genau dann einer „Orientierung" bedarf, wenn es sich auf Bereiche erstreckt, die jenseits des Wißbaren liegen, bei denen es aber nicht möglich ist, Urteilsenthaltung zu üben, weil ein „Bedürfnis der Vernunft" zum Urteilen treibt. 'Ein „Orientierungswissen "2 also ist für Kant begrifflich ausgeschlossen. Allerdings legt er seinen speziellen, erfahrungsgebundenen Wissensbegriff zugrunde. Außerdem hat Kant emphatisch betont, daß der Maßstab der „Orientierung" nur in der Vernunft selbst liegen kann und daß ihr Resultat dem Wissen insoweit ähnlich ist, als es mit diesem zwar nicht die „Gewißheit“, wohl aber die „Festigkeit" und das „Bewußtsein seiner Unveränderlichkeit" teilt. ${ }^{3}$

Ohne mich an die speziellen Prämissen Kants zu binden, möchte ich im folgenden den Versuch machen, seine Frage nach der „Orientierung im Denken“ in sinngemäß modifizierter Form auf das Wollen zu übertragen. Auch hier, so meine ich, gibt es einen Bereich, der prinzipiell über die Erfahrung hinausführt und der es zweifelhaft macht, ob oder in welchem Sinne wir überhaupt noch von "Wissen" reden können. Verschärft wird dieser Zweifel dadurch, daß die „Orientierung im Wollen“" nicht nur in gravierende theoretische Fragen führt, sondern auch und zuallererst, wie ich zeigen will, in spezifisch praktische. Hier geht es nicht allein darum, ein "festes“ (wenn vielleicht auch

I. Kant, Was heißt: Sich im Denken orientieren? (Oktober 1786), zit. nach AA, Bd. VIII, 133-147.

2 "Orientierungswissen" wird in der neueren Literatur meist kontrastiv zu rein theoretischem "Tatsachenwissen", teils auch zu zweckrationalem (technischem) „Verfügungswissen" gebraucht und bezieht sich speziell auf die Orientierung an universalen, höchstrangigen Werten und Zwecken. Zum Sprachgebrauch vgl. G. Wolters, Orientierungswissen als Humanressource, in: G. Clar / J. Doré / H. Mohr (Hrsg.), Grundlagen einer nachhaltigen Entwicklung, Berlin 1997, 33-51. Die Rede vom „Wissen“ ist problematisch, läßt sich jedoch verständlich machen, wenn man, wie im folgenden gezeigt werden soll, einen rein theoretischen und einen spezifisch praktischen, volitionalen Sinn von "Wissen"scharf auseinanderhält.

3 Kant, a.a.O., 141 f., Anm. 
nicht "gewisses") Urteil darüber zu bekommen, was ist, sondern ein „festes" (oder doch hinreichend tragfähiges) Urteil darüber, was sein soll.

\section{Relevanz theoretischen Wissens}

Die Rede vom „Orientieren" hat metaphorische Wurzeln. Exemplarisch sind Situationen, in denen ein Wanderer oder Schiffer die Orientierung verloren hat und deshalb hilflos umherirrt. Ein Kompaß kann ihn darüber unterrichten, wo Orient und Okzident liegen. Aber das allein hilft ihm nicht weiter. Er muß auch über eine verläßliche Karte oder entsprechende räumliche Vorstellung von seiner Umgebung verfügen und wissen, wo er sich gerade befindet. Mit diesen Informationen (nehmen wir an) ist er theoretisch hinreichend orientiert, d.h. er kennt alle ihn interessierenden Fakten. Normalerweise hilft ihm das, aber natürlich nur, wenn er weiß, wo er hin will. Weiß er es nicht, bleibt er praktisch desorientiert. Denn weil er nicht weiß, was er will, weiß er auch nicht, was er tun soll, und wird deshalb genauso ratlos umherirren oder am Fleck verharren wie zuvor. Wenn er aus dieser Lage herauskommen will, muß er praktische Überlegungen anstellen, die eine doppelte Orientierungsfunktion für ihn erfüllen: Sie dienen zunächst der Willensbildung und bereiten über diese den nachfolgenden Entschluß zum willensgemäßen Handeln vor. In einfachen Fällen sind beide Schritte kaum voneinander zu trennen. Aber auch hier ist die Willensbildung sachlich das Erste. Ihr speziell dient die "Orientierung im Wollen". Und die Frage ist nun, was dies genau beinhaltet und ob es gerechtfertigt ist, auch hier (ähnlich wie bei rein theoretischen, faktenbezogenen Überlegungen und Erkundungen) von einem Erwerb von Wissen zu sprechen.

Nun, zu einem bedeutenden Teil zumindest hängt auch unsere Willensbildung von erworbenem Wissen ab. Denn sie bedarf der Kenntnis von Fakten. Dabei geht es zunächst um mögliche Willensinhalte. Wenn ich nicht weiß, welche Speisen ein Restaurant anbietet oder welche Sehenswürdigkeiten in einer fremden Stadt zu besichtigen sind, kann ich mir auch nicht darüber klar werden, ob oder welche von ihnen ich vielleicht essen oder besichtigen will.

Sodann dienen willensbildende Überlegungen dazu, herauszufinden, ob oder unter welchen Bedingungen mögliche Willensinhalte realisierbar sind. Dabei wird häufig nur an die Erkenntnis von Mitteln gedacht, die man einsetzen kann oder muß, um bestimmte Inhalte als $Z$ weck zu erreichen. Natürlich bilden diese einen besonders wichtigen Teil. Aber es wäre mehr als kurzsichtig, seine Willensbildung nicht auch auf die Kenntnis der Folgen und Nebenfolgen zu stützen. Die blind euphorische Entscheidung der Industriestaaten, ihre Energieprobleme durch expansive Nutzung der Kernenergie zu lösen, ohne sich um die Entsorgung des strahlenden Mülls zu kümmern, liefert dafür das wohl verhängnisvollste Negativbeispiel. Alle realisierbaren Willensinhalte sind vielfältig eingebettet in ein komplexes Netz von Bedingungen, ohne die sie nicht zu verwirklichen sind. Nur diese Komplexe, nicht die isolierten Einzelinhalte, bilden die "Optionen", zwischen denen man realistisch wählen kann. Und da die Anzahl der Folgen, Nebenfolgen und Mittelglieder zwischen primärer Handlung und gewolltem Erfolg indefinit, also prinzipiell unüberschaubar ist, stoßen wir hier bereits auf einen Bereich, der die Gren- 
zen unserer Erfahrung sprengt. Dieser Tatsache muß man Rechnung tragen, auch wenn viele Theoretiker sie verdrängen. Die weit verbreitete, aber konzeptionell verwirtte Entgegensetzung von "Gesinnungs" und "Verantwortungsethik", "deontologischen" und "konsequentialistischen" Moraltheorien belegt das.

Das Netz der Bedingungen, die Optionen konstituieren, wird keineswegs nur durch kausale oder naturgesetzliche Relationen geknüpft, sondern auch durch diverse andere, z.B. geltende soziale Regeln. Deshalb müssen sich realistisch willensbildende Überlegungen auch an Normen und Werten orientieren. Obwohl diese selbst nicht deskriptiv sind, sondern präskriptiv, stellt ihre Ermittlung eine theoretische Aufgabe dar, deren Ergebnis "Wissen" ist. Das deutsche Strafrecht bringt das korrekt zum Ausdruck, wenn es außer vom ,Tatbestandsirtum" auch vom ,Verbotsirrtum" spricht. Objekt des Wissens oder Nichtwissens ist ja nicht das Verbot als solches, sondern die Tatsache, daß es in Kraft ist und erfüllungsabhängige Konsequenzen hat. Wer das Sonderangebot eines Kaufhauses nutzen will, tut gut daran, nicht nur zu wissen, wohin er gehen muß und in welcher Zeit, sondem auch, daß er das, was er will, nur kriegen kann, wenn er entweder zahlt oder straffällig wird.

Insoweit also ist die Rede von einem ,Orientierungswissen“" auch mit Bezug auf die Orientierung im Wollen verständlich. Allerdings darf man zwei Dinge nicht aus den Augen verlieren. Erstens ist dieses „Wissen“ ausschlieBlich theoretisches Wissen, das in einem sicheren Urteil darüber besteht, was ist, einschließlich faktisch erhobener Sollensansprüche. Zweitens ist es durchweg begrenzt, erstreckt sich also niemals auf alles, was Gegenstand unseres Wollens werden kann.

\section{Spezifisch praktisches Wissen?}

Dieser zweite Punkt zeigt bereits, daß theoretisches Wissen zwar notwendig, nicht aber hinreichend für eine realistische Willensbildung ist. Daß eine bestimmte Norm in einer Gesellschaft gültig ist, kann man als distanzierter Beobachter auch feststellen, ohne sich selbst durch sie verpflichtet zu fühlen. Und wenn man sich verpflichtet fühlt, heißt das nicht unbedingt, daß man will, was die Norm fordert. Im Gegenteil, es gehört zum Sinn normativer Verhaltenssteuerung, daß sie dem Adressaten die Freiheit läßt, sich für oder gegen sie zu entscheiden. Noch deutlicher ist die Distanz bei nichtnormativen Fakten. Auch wenn ich weiß, welche Speisen ich realistischerweise ordern oder welche Sehenswürdigkeiten ich auf welchem Wege ansteuern kann, ist meine Willensbildung damit nicht abgeschlossen. In gewissem Sinne beginnt sie sogar jetzt erst. Denn der entscheidende Schritt wird durch die Optionenermittlung nur vorbereitet. Theoretisches Wissen bezieht sich eben nur auf das, was ist, Wollen aber auf etwas, das (in den Augen des Wollenden) sein soll.

"Wollen" ist mehr als bloßes Wünschen, enthält aber Wünschen als begrifflichen Kern. Und dieses ,Wünschen " ist seinerseits im Kern nichts anderes als eine (der assertorischen spiegelbildlich entgegengesetzte) optativische Stellungnahme, mit der der Anspruch erhoben wird, etwas möge der Fall sein. Daß dies so ist und daß Einstellungen des Wollens und Wünschens sich weder hedonistisch noch dispositionell reduzieren las- 
sen, dafür habe ich andernorts ausführlich argumentiert. ${ }^{1}$ Ebenso werde ich nicht wiederholen, warum ich überzeugt bin, daß optativische Einstellungen primär nur als Bewußtseinszustände identifizierbar sind, ${ }^{2}$ ohne daß dies die Möglichkeit einer sekundären physikalistischen Reduktion ausschließt. Allerdings ist eine solche Reduktion (trotz der euphorischen Aufgeregtheiten mancher KI- und Neuro-Freaks) bis auf weiteres reine „science fiction". Etwas zu wollen also heißt primär, etwas bewußt zu wünschen und ebendamit den Anspruch zu erheben, daß es sein soll, nicht jedoch, zu behaupten, daß es so ist.

Gewiß, es gibt Fälle von Sollensansprüchen, die wir zugleich als erfüllt erkennen, z.B. wenn etwas so gekommen ist, wie wir wollten und weiterhin wollen. Doch überall, wo es nicht so ist, reicht unser Wille zwar nicht unbedingt über alles Erfahrbare, wohl aber über unsere Erfahrung hinaus. Damit jedoch wird die Vorstellung von der „Orientierung im Wollen" als Wissenserwerb in doppelter Hinsicht fragwürdig. Zweifelhaft ist sie nicht nur im Blick auf den Schritt von der bloßen Optionenerkenntnis zur Ausbildung eines bestimmten Wollens. Zweifelhaft ist sie auch für den Zustand des Wollens selbst. Denn macht es Sinn, eine nicht assertorische, rein optativische Einstellung als „Wissen" anzusprechen?

Nun, es gibt etablierte Redeformen, die zweifellos nichtassertorisch sind. Eine davon ist die Rede vom ,Wissen wie ". Doch sie kann unser Problem nicht lösen. Denn einerseits ist dieses meist auch mit assertorischem „Wissen daß" verbunden. Andererseits lassen sich nur bestimmte, relativ simple Formen des Schrittes vom Wollen des Zwecks zum Wollen und Ergreifen des Mittels als Fälle von „Wissen wie“ verstehen, nicht aber andere Arten der Willensbildung, geschweige denn das Wollen selbst. Nur eine andere nichtassertorische Redeform könnte uns weiterhelfen.

Von Menschen, die in ihrem Wollen desorientiert sind, sagen wir auch, sie ,wüßten nicht, was sie wollen", von Menschen, die orientiert sind, sie "wüßten es". Was aber heißt das? Zunächst wohl nur, daß sie sich ihres Willens bewußt sind, unterschieden von Zuständen des unbewußten, verdrängten oder nur halb bewußten Wollens. Dann aber kann es auch heißen, daß ihre Willensbildung beendet ist und sie zu einem bestimmten, festen Wollen gekommen sind. In diesem Sinne von "Wissen“ ist die Beschreibung des Willensbildungsprozesses als Wissenserwerb offenbar unanfechtbar. Allerdings scheint das ein Sinn zu sein, der sich vom theoretischen, faktenbezogenen Wissen fundamental unterscheidet. Oder nicht? Verbirgt sich hinter der scheinbar anders gearteten Rede davon, daß jemand „weiß, was er will“", vielleicht nur die Einsicht, daß auch die „Orientierung im Wollen" sich letztich theoretischem Wissen verdankt, wenn auch eines Wissens, das anderer Art ist als die bloße Optionenkenntnis?

\section{Ergebnisoffene und nicht ergebnisoffene Überlegungen}

Um diese Frage beantworten zu können, müssen wir die Willensbildung näher betrachten. Manchmal bildet sich unser Wollen blitzartig oder überfallartig. Ein plötzlicher

\footnotetext{
G. Seebaß, Wollen, Frankfurt 1993, vgl. bes. 47, 72, $91 \mathrm{ff} ., 113 \mathrm{f}, 168 \mathrm{f}$.

Vgl. Seebai 1993, a.a.O., 46f, 66ff.
} 
Schmerz, eine plötzlich auftauchende Gefahr erregen sofort den Willen in uns, dem zu entkommen. Oder ein Objekt, auf das wir beim Stöbern in einer Kunsthandlung stoßen, entringt uns den Ausruf: „Das will ich!“. Doch das sind eher seltene Fälle. Im allgemeinen bedarf es zur Überwindung von Zuständen anfänglicher volitionaler Desorientiertheit eines gewissen Maßes an Überlegung - praktischer Überlegung natürlich, die zuerst darauf zielt (192), uns über unser Wollen klar zu werden. Was genau ist darin involviert?

Zwei Formen willensbildender Überlegungen sind auseinanderzuhalten: ergebnisoffene und nicht ergebnisoffene. Letztere zeichnen sich dadurch aus, daß das, was der Überlegende will, schon am Beginn seiner Überlegungen feststeht. Warum aber überlegt er dann noch? Nun, einiges ist auch hier zunächst unklar. Auch wer schon weiß, was er will, muß das Geschäft der Optionenermittlung betreiben. Denn er muß nicht nur feststellen, wie er das Gewollte realisieren kann, sondern auch und vor allem, ob unter den relevanten Mitteln, Folgen und Nebenfolgen einige sind, die sich mit etwas, das er ebenfalls und vielleicht stärker will, nicht vereinbaren lassen. Außerdem ist uns beileibe nicht alles, was wir wollen, immer präsent, sondern muß erst bewußt gemacht werden. Dazu ist das Durchdenken der Bedingungen, in die ein bestimmter Willensinhalt optional eingebettet ist, eines der wichtigsten heuristischen Hilfsmittel. Aber natürlich kann man auch direkt auf seine komplexere Willenslage reflektieren oder andere Hilfen in Anspruch nehmen, bis hin zur extensiven psychoanalytischen Therapie. Wenn wir nun annehmen, daß es bei all dem nur um die Aufdeckung von etwas geht, das bereits da ist, und da $\beta$ das Resultat durch die ebenfalls schon vorhandenen Präferenzen zwischen den einzelnen Willensinhalten eindeutig festgelegt ist, müssen wir sagen, daß der gesamte Überlegungsprozeß, was das Wollen betrifft, von vorneherein nicht ergebnisoffen war. Klärungsbedürftig war hier nur, was alles man immer schon wollte und jeweils in welchem Maß. Und die Beantwortung dieser Frage ist offenbar ebenso eine Sache des theoretischen Wissenserwerbs wie die rein theoretische Optionenermittlung.

Noch ein anderer Aspekt nicht ergebnisoffener Überlegungen muß erwähnt werden. Auch eine Person, die „weiß, was sie will“, weiß nicht immer, warum. Sie „orientiert sich im Wollen", indem sie die Gründe ermittelt, die ihm vorausliegen. Das können Kausalgründe sein oder auch (rein ${ }^{1}$ ) rationale Gründe, wie etwa der übergeordnete Wille, bestimmten Normen genüge zu tun. Unter der Annahme, daß auch diese Gründe bereits bestehen und nur noch aufgedeckt werden müssen, läuft auch dieser Orientierungsprozeß auf ein volitional nicht mehr ergebnisoffenes Überlegen hinaus, das durch theoretischen Wissenserwerb beendet wird.

Im Unterschied dazu sind ergebnisoffene Überlegungen solche, bei denen die entscheidende optativische Stellungnahme erst noch erfolgen muß und durch willensbildendes Überlegen ermöglicht wird. Wird hier nach relevanten Gründen gefragt, so geht es immer um rationale Gründe, und es steht nicht von vorneherein fest, welche von ih-

1 Rationale Begründungszusammenhänge lassen sich nicht einfach auf kausale reduzieren. Aber das schließt nicht aus, anders als manche Vertreter der Analytischen Philosophie in den 50er und 60er Jahren dachten. daß Menschen durch rationale Gründe kausal motiviert sein können. Im GegenteiI, persönlich zurechenbar sind willentliche Handlungen nur, wenn sie sich auch kausal auf den Willen des Handelnden zurückführen lassen. Näheres dazu in Seebaß 1993, a.a.O., $205 f f$. 
nen den Ausschlag geben. Die überlegende Person will sich ihrer theoretisch versichern, um danach in optativischer, nichtassertorischer Form zu ihnen Stellung zu nehmen. Entsprechendes gilt für die Optionenermittlung und die Vergewisserung über die zunächst verdeckte, komplexe Vielfalt des eigenen Wollens. Auch hier dient die theoretische Wissenserweiterung nicht dazu, neugierig in Erfahrung zu bringen, was eigentlich längst vorentschieden ist, sondern den Spielraum der faktischen und volitiven Möglichkeiten kennenzulernen, die man besitzt, um daraufhin Stellung zu nehmen. Weiß der Überlegende danach, was er will, so hat dieses „Wissen“ zwar einen bedeutenden, mehr oder weniger weitreichenden theoretischen Hintergrund, geht aber darin nicht auf.

\section{Vorzüge mangelnder Ergebnisoffenheit}

Der Gedanke, daß rationale Akteure sich ausschließlich von Überlegungen leiten lassen, die nur dem theoretischen Wissenserwerb verpflichtet und volitiv nicht mehr ergebnisoffen sind, kann faszinieren. Wenn feststeht, was die Individuen wollen und wie ihre real (,optional") unvereinbaren Willensinhalte präferentiell geordnet sind, wird der Prozeß ihrer „Orientierung im Wollen" berechenbar. Man kann versuchen, Logiken des praktischen Überlegens zu formulieren, mit Hilfe derer sich, angewandt auf bestimmte epistemische und volitive Prämissen, rationale Empfehlungen zum Wollen und Handeln ableiten lassen. Soweit die Betreffenden rational sind, werden sie sich entsprechend verhalten. Und überall, wo sie es nicht sind, kann man versuchen, die Gründe für ihr Abweichen vom rationalen Ideal aufzudecken und ihr Verhalten darüber wieder berechenbar machen. Soziale Koordinations- und Integrationsprobleme z.B. könnten auf diesem Wege lösbar werden. Es ist deshalb nicht verwunderlich, daß Varianten des nicht ergebnisoffenen Überlegungsmodells vor allem für Psychologen, Sozialwissenschaftler und Ökonomen, aber auch für viele Philosophen attraktiv sind.

Wichtig erscheint das Modell aber auch außerhalb des sozialen Kontexts. Denn zwei Funktionen, die auch für Individuen essentiell sind, können offenbar nur durch die mangelnde Offenheit praktischer Überlegungen sichergestellt werden. Erstens erfüllt sie eine Entlastungsfunktion. Würden wir etwa bei jedem Schritt, den wir tun, neu dazu Stellung beziehen müssen, ob oder welche der uns verfügbaren Optionen wir wollen, würden wir alsbald handlungsunfähig. Einmal gefaßte Pläne und Vorsätze könnten wir nicht konsequent zu Ende führen. Außerdem hätten wir ständig neu zu entscheiden, $o b$ wir die Optionenermittlung abbrechen oder noch weiter vorantreiben. Permanentes Infragestellen aber hindert uns eher, als daß es uns hilft. Faktisch sind wir zum Glück nicht so desorientiert, daß permanente Überlegungen nötig wären. Ein großer Teil unseres Alltagslebens vollzieht sich sogar völlig habitualisiert und automatisiert. ${ }^{\prime}$ Und wenn Willensentscheidungen fällig sind oder automatisierte Prozesse reflexiv überdacht werden, genügt meist die theoretische Vergewisserung über die schon bestehenden Willensinhalte und Präferenzen.

' Neuere motivationspsychologische Untersuchungen liefern dazu vielfältiges Material. Vgl. etwa J.A. Bargh, The Automaticity of Everyday Life, in: R.S. Wyer (ed.), The Automaticity of Everyday Life. Advances in Social Cognition, vol. X, Mahwah/N.J. 1997, 1-61. 
Zweitens dient fehlende volitionale Offenheit einer umfassenden, einheitlichen Lebensorientierung. Wer sich nicht länger orientieren muß, sondern weiß, was er will, weiß damit in gewissem Sinn auch erst, wer er ist. Durch sein fixiertes Wollen gewinnt er Selbstidentität, Selbstsicherheit und innere Freiheit. Daß dies so ist und wie man dahin gelangen kann, ist ein altes Thema unserer Geistesgeschichte. Im Aristotelischen Konzept eines „tugendhaft festen Charakters“ läßt es sich ebenso ausmachen wie im Stoischer, Gedanken der ,Ataraxie* oder der jüdisch-christlichen Vorstellung vom Menschen, der erst in der vollständigen Bindung des Willens an Gott sein wahres Selbst gewinnt. Kollektivistische Versionen finden sich in der Neuzeit bei Hegel und Marx ${ }^{1}$ und in gewissem Sinn auch in Kants Vernunftmoral. ${ }^{2}$ Individualistisch verschärft kehrt das Thema auch in der Existenzphilosophie wieder. Und in der neueren Literatur ist es vor allem Harry Frankfurt gewesen, der den konstitutiven Zusammenhang zwischen Selbstsein und Vergewisserung über das eigene Wollen herausgestellt hat, bis hin zu der Behauptung, daß bestimmte Formen ,volitionaler Notwendigkeit" kriteriell dafür seien, was uns als Personen ausmacht."

Ob man so weit gehen kann, ohne die für unser Selbstsein indispensible Autonomie aufs Spiel zu setzen, mag hier noch dahin gestellt bleiben (vgl. dann $206 \mathrm{ff}$.). ${ }^{4}$ Klar ist

1 G.W. Hegel, Grundlinien der Philosophie des Rechts, Einleitung; Enzyclopädie, $\$ 469$ ff; K. Marx, Zur Judenfrage, in: K. Marx / F. Engels Werke, Bd. I, Berlin 1970, 361-370; ders., Die deutsche Ideologie, in: a.a.O., Bd. III, Berlin 1983, 74-77.

2 Das gilt speziell im Hinblick auf jene Passagen, in denen Kant das (allein ,achtungswürdige“" „Personsein" menschlicher Individuen vollständig - unter Ausschluß aller partikulären Neigungen und sonstigen empirisch-psychologischen Eigenschaften - auf jene moralisch-praktische Vernünftigkeit reduziert, die sie mit allen anderen Menschen bzw. allen vernünftigen Wesen teilen. Vgl, dazu insbesondere I. Kant, Grundlegung zur Metaphysik der Sitten, AA, Bd. IV, 401 Anm., 428f,, 434f., 457f.; ders., Kritik der praktischen Vernunft, AA. Bd. V, 73, 76-78, 81 Anm; ders., Die Metaphysik der Sitten, AA, Bd. VI, 223, 418, 420, 434-436, 447, 449f., 462-464. Der, rationalistische Kollektivismus" Kants, der die menschliche Individualität ebenso wie seine Sinnlichkeit eskamotiert, ist m.E. auch der Kern der Kritik, die Schiller zu Recht an ihm geübt hat.

3 H. Frankfurt, The Importance of What we Care About, Cambridge 1988; Necessity, Volition, and Love, Cambridge 1999. Vgl. zur „volitional necessity“ speziell 1988, 85ff. und 1999, $110 \mathrm{ff}$. sowie programmatisch $1999, \mathrm{X}$.

4 Einschlägige Argumente gegen Frankfurts Konzept der „volitionalen Notwendigkeit" findet man z.B. bei E. Tugendhat, Philosophische Aufsaatze, Frankfurt 1992, 464-467. Tugendhats (auch nur mit Vorbehalt vorgetragener) Verdacht des Aristotelischen Essentialismus trifft Frankfurt allerdings nicht, denn dessen Rede von der „essential nature of a person“ $(1999,113 \mathrm{f}$.) beinhaltet offenbar nicht mehr als jene volitionalen Eigenschaften, ohne die eine Person de facto nicht diejenige wäre, die sie ist, fernab jeden Gedankens an eine vorgegebene Seinsordnung oder verdeckte Entelechien. Auch der Einwand einer Vermischung von (äußerem oder innerem) Zwang, der Freiheit und Autonomie beeinträchtigt, und einem in dieser Beziehung prinzipiell harmlosem Nicht-anders-wollenKönnen erseheint mir unberechtigt. Frankfurt bestreitet ja keineswegs, daß Entscheidungen, die durch ein „Wollen-Müssen“ (,volitional necessity") eingeschränkt sind, sehr wohl autonom und frei sein können, sondern behauptet sogar im Gegenteil, daß sie es nur unter dieser Bedingung sind (vgl. 1999, 110, 114).

Verwirrend an Frankfurts Konzept ist seine mehrdeutige Rede nicht, wie Tugendhat meint, von der „Notwendigkeit", sondern vielmehr von der ,Freiheit des Wollens", aufgefaßt als Freiheit der Wil- 
jedoch, daß beide erwähnten Funktionen keinen Beweis dafür liefern, daß volitional ergebnisoffene Überlegungen keinerlei Anteil an unserer „Orientierung im Wollen“ haben. Gewiß, lebensbestimmend können auch Willensinhalte sein, die uns genetisch, traditional oder individuell sozialisatorisch vorgegeben sind. Ebenso signifikant aber (und in der Literatur am meisten beachtet) sind Situationen, in denen ein Mensch sich bewußt für eine bestimmte Lebensorientierung entscheidet, z.B. für ein moralisches, religiöses oder politisches Ideal oder auch nur für den eigenen Beruf oder das Leben mit einem Partner. Noch deutlicher ist das bei der Entlastungsfunktion. Daß wir bestimmte Arbeitsabläufe oder längere Handlungssequenzen unreflektiert oder völlig automatisiert ausführen, schließt die bewußte, überlegte Entscheidung für sie nicht aus. Ja, sie beruht im Normalfall auf einer solchen, ohne daß feststünde, daß daran keine ergebnisoffenen Überlegungen beteiligt sind. Und das Interesse von Wissenschaftlern an berechenbaren, präferentiell geordneten Wunsch- und Willenshaltungen ist allemal kein Beweis, daß

lensbildung. Nur in einem bestimmten Sinne von „Freiheit" hält Frankfurt sie für vereinbar mit Autonomie. In einem anderen Sinne soll sie es nicht sein, verstanden nämlich als Fähigkeit, beliebige Inhalte bindungslos zum Gegenstand seines Wollens zu machen (vgl. 1999, 108, 109f.). Ein derart „freier Mensch" kann, so scheint es, nicht autonom sein, weil er volitional offenbar völlig desorientiert ist und damit auch kein (volitional gedeutetes) Selbst besitzt.

Dieser Freiheitsbegriff erinnert an den Begriff der ,negativen Freiheit' bei Erich Fromm, Die Furcht vor der Freiheit, Berlin 1983, vgl. bes. 30ff., 204ff. Wie Frankfurt so diagnostiziert auch Fromm einen stetigen Zuwachs, den die ,negative Freiheit" in der Neuzeit erfahren habe, und einen damit verbundenen drohenden Verlust an Selbstgewißheit und Sicherheit. Allerdings sieht er darin einen unvermeidlichen Ausdruck der conditio humana (a.a.O., 31f. 189f.), wenngleich keinen erschöpfenden. Die Kehrseite der auf sich allein gestellten „Freiheit von jeder Bindung“ sei jene ,panikartige Flucht vor der Freiheit in neue Bindungen" (a.a.O., 35), die im 20. Jh. zur autoritären, konformistischen Unterwerfung unter den Totalitarismus geführt habe (a.a.O., Kap. 5-6). Selbstheit und Individualität gewinne man erst. wenn die "negative“ durch eine ,positive Freiheit" ergänzt werde, die ,im spontanen Tätigsein [activity] der gesamten, integrierten Persönlichkeit" besteht (a.a.0., 205, vgl. 33, 207ff.). Damit hat Fromm, so scheint mir, das Wesentliche getroffen, während Frankfurt gerade hier lückenhaft bleibt und damit den Hauptgrund für Zweifel an seinem Autonomiekonzept liefert:

"Volitionale Notwendigkeit" kann schließlich auch Ausdruck genetischer oder sozialer Determiniertheit, Borniertheit oder gelungener „Flucht vor der Freiheit" sein. Ausdruck von Autonomie und (,positiver") Freiheit ist sie nur, wenn sie autonom entwickelt wurde und autonom beibehalten wird. Dann aber ist sie nicht mehr im wörtlichen Sinne "notwendig" (vgl. auch Tugendhat a.a. $0 ., 466)$. Denn damit würde zu ihr auch das Bewußtsein gehören, daß die bestehende "Orientierung im Wollen" problematisierbar ist und durch eine andere ersetzt werden könnte, mag die betreffende Person sich bis auf weiteres auch bewußt dagegen entscheiden. Frankfurt schließt Veränderungen nicht aus (vgl. 1999, 112, $115 \mathrm{f}$ ), hat aber kaum etwas in der Hand, um autonome von nicht autonomen Änderungen zu unterscheiden. Fromms Kriterium der ,Aktivität" ist mit der Rede von „volitionaler Notwendigkeit" jedenfalls kaum zu vereinbaren, und es ist zweifelhaft, ob Frankfurt ihm genüge tun kann, obwohl er es möchte (vgl. 209, Anm. 2, 210, Anm.1). Hat ein Mensch, der ,nicht anders wollen kann", wirklich ein freies "Selbst " $\left(\right.$ self $\left.f^{\prime \prime}\right)$ damit gewonnen? Ich glaube nicht. Auch Frankfurts wiederholter Vergleich dieses Zustands mit dem der Liebe (z.B. 1988, 89f., 94; 1999, 106, 114) ist hier verräterisch, charakterisiert er diese doch selbst verständlicherweise, aber desaströs für seine These - gerade als einen Zustand der , selflessness" $(1988,89 ; 1999,114)$. 
die menschliche Wirklichkeit dem entspricht. Wenn man Willensbildungsprozesse durch spezifisch praktische, ergebnisoffene Überlegungen generell ausschließen will, muß man stärkere Argumente dafür ins Feld führen. Welche könnten dies sein?

\section{Unzureichende Argumente für mangelnde Ergebnisoffenheit}

Das historisch einflußreichste Argument wird meist auf Aristoteles, teils auch auf Hume zurückgefuhrt und besagt, daß praktische Überlegungen prinzipiell zweckrationale seien, sich also nur auf die Suche nach Mitteln zu gegebenen Zwecken beziehen könnten.' Doch in dieser unqualifizierten Form ist die Behauptung keinesfalls haltbar. Wenigstens zwei Ergänzungen muß man vornehmen. Erstens muß man neben den Mitteln auch die Folgen und Nebenfolgen berücksichtigen, d.h. die optionale Willensbildung im ganzen (192). Zweitens muß man auch jene Überlegungen einbeziehen, die der Vergewisserung über die eigenen Zwecke dienen. Auch mit diesen Ergänzungen jedoch ist die These unhaltbar.

Auch bei hochrangigen Zwecken, die Anspruch darauf erheben können, Orientierungen für das gesamte Leben zu sein, kann man nicht ausschließen, daß mehrere gleichgewichtig nebeneinanderstehen. Frankfurt hat dies als das Problem der volitionalen "Ambivalenz" oder „Halbherzigkeit" beschrieben. ${ }^{2}$ Präferentielle Eindeutigkeit wäre hier prinzipiell nur zu gewährleisten, wenn man annehmen könnte, daß es einen und nur einen obersten Zweck gibt, dem alle anderen sich rational fugen. Das hat man immer wieder zu zeigen versucht. Doch weder das Aristotelische Streben nach einem ,bios theoretikós" noch das Freudianische „Lustprinzip" oder das „unruhige Herz" des Augustinus, das erst in Gott seine Ruhe findet, ${ }^{3}$ sind sehr plausible Kandidaten für einen obersten Zweck, den alle Menschen verfolgen. Nicht einmal das Streben nach Selbsterhaltung, Hobbes" Universalprinzip, kann uneingeschränkt Anspruch darauf erheben, wie eine Vielzahl von Selbsttötungen und lebensgefährlichen Unternehmungen zeigt. Formalbegriffe des „Guten" jedoch oder Begriffe wie "Nutzen", „Glück" oder "Lust" im abstrakten Sinne können als inhaltsleere Prinzipien überhaupt keine Orientierungsfunktion ubernehmen. Kant hat das in seiner Eudaimonismuskritik klar herausgearbeitet. ${ }^{4}$ Annehmbar wäre allenfalls, daß jedes Individuum seinen eigenen Universalzweck verfolgt. Aber auch das ist empirisch implausibel. Und selbst wenn es so wäre, ließe sich doch nicht ausschließen, daß auch dieser Zweck irgendwann einmal zum Gegen-

Vgl. Aristoteles, Ethica Nicomachea 1112b11-30; Ethica Eudemica 1226b10-17, 1227a6-13, 1227b22-1228a1; Metaphysica 1032b1-14; Hume, Treatise 11. 3,3; 1I1, 1,1. Ob Aristoteles diese Radikalposition tatsächlich vertreten wollte, ist zweifelhaft, kann hier aber offen bleiben. Vgl, dazu H. Steinfath, Orientierung am Guten. Praktisches Überlegen und die Konstitution von Personen, Habilitationsschrift Konstanz I999, Kap. 1.4, Anm. 34 und Kap. 2.2.

Vgl. insbesondere Frankfurt 1988, a.a.O., 66-68, 159ff; 1999, a.a.0., 98ff.

Augustinus, Confessiones I, 1,1.

4 Vgl. speziell Grundlegung zur Metaphysik der Sitten, AA Bd. IV, 418; Kritik der praktischen Vermunft, AA Bd. $V, 25,36$. 
stand einer psychoanalytischen oder radikalen Cartesianischen Selbstüberprüfung gemacht wird.

Hinzukommt, daß auch bei zweckrationalen Überlegungen all diese Probleme wiederkehren. Es läßt sich eben nicht ausschließen, sondern ist im Gegenteil ubiquitäre Alltagserfahrung, daß mehrere gleichgute Mittel verfügbar sind, daß vorhandene Gütedifferenzen durch die Realisierungswahrscheinlichkeiten aufgewogen werden oder daß ein präferentielles Übergewicht durch die „Kosten“ bei Folgen und Nebenfolgen konterkariert wird. Manche Philosophen - Leibniz vor allem - haben deshalb zu zeigen versucht, daß ein präferentielles Gleichgewicht real unmöglich sei. ${ }^{1}$ Doch das ist philosophisches Wunschdenken. Empirisch wäre es allenfalls durch extensiven Rekurs auf unbewußte Präferenzen verständlich zu machen. Solche kann man nicht generell ausschließen. Die Beweislast für ihr Bestehen im Einzelfall liegt aber klarerweise bei ihren Vertretern. ${ }^{2}$

Anerkennt man Entscheidungen bei präferentiellem Gleichgewicht kann man allerdings immer noch sagen, daß diese nicht in Abhängigkeit von rationalen, volitional ergebnisoffenen Überlegungen fallen, sondern durch Zufall. Das ist die klassische Lösung für Wahlsituationen nach dem Modell von „Buridans Esel". ${ }^{3}$ Aber ist sie die einzig mögliche und beweist sie, was sie beweisen soll? Das bloße Abwarten und Nichtstun hat selten den Sinn, das Ergebnis (wie man so sagt) "dem Zufall zu überlassen". Meist ist es eine Entscheidung für eine der Alternativen, nur eben durch Unterlassen. Man braucht schon die Intervention eines Zufallsgenerators, z.B. eine geworfene Münze. Doch wenn diese gefallen ist, steht man immer noch vor der Entscheidung, ob man dem Orakel nun weiterhin folgen soll oder nicht. Und auch wenn der Zufallsgenerator unmittelbar wirksam wird, bleibt die Entscheidung für das Verfahren als solches. Die klassische Lösung unterstellt hier die Existenz eines übergeordneten Wollens, z.B. den Wunsch eines anthropomorphen Esels, nicht zu verhungern, egal auf welchem konkreten Wege. Doch das läuft wieder auf jenes Postulat vorgegebener Zwecke hinaus, das wir nicht akzeptieren können. Außerdem muß man sich ja selbst dann für den Einsatz des Zufalls als Mittel entscheiden. Und ist es so klar, daß dieser Entscheidung keine anfänglich ergebnisoffene Überlegung vorausgeht?

I Vgl. z.B. G.W. Leibniz, Confessio Philosophi, hrsg. v. O. Saame, Frankfurt 1967, 78ff.; Nouveaux Essais I, 1, 15; II. 21, 17-19, 36, 47-48; ders., Theodizee $\$ 35, \$ 45-51$; Briefe an Clarke IV,1-6; V, 15-17. Diverse Belege aus anderen Autoren bietet N. Rescher, Choice without Preference, in: KantStudien 51 (1959-60), 142-175.

2 Deshalb hat Leibniz sich auch nicht nur auf empirische Belege für seine These verlassen wollen, sondern den metaphysischen "Satz vom Grund" bemüht, um die Annahme unbewußt präferenzierender Faktoren überall dort plausibel zu machen, wo sie empirisch nicht aufweisbar sind. De facto ist das ein Eingeständnis, daß nicht die Phänomene diesen Gedanken erzwingen, sondern das Interesse des Philosophen an einer durchweg geordneten, berechenbaren Welt.

3 Vgl. z.B. P. Bayle, Dictionnaire, Art. ,Buridan" C; A. Schopenhauer, Der handschrifliche Nachlaß, Bd. I, Frankfurt 1966, 328f.; Rescher 1959-60, a.a.O., 167ff. 


\section{Optional willensbildende Überlegungen}

Diese letztere Frage zwingt uns, unser Problem noch etwas tiefer anzusetzen. Angenommen einmal, willensbildende Überlegungen träten tatsächlich nur in der Form auf, daß ein vorhandenes Wollen durch die theoretische Optionenermittlung ergänzt wird. Wie genau vollzieht sich dann der Schritt zu einem subordinierten Wollen, also z.B. beim zweckrationalen Schließen? Handelt es sich um einen regelgeleiteten Akt, ähnlich rein theoretischen Schlußfolgerungen ,salva veritate" oder regelgerechten Zügen beim Schach? Oder handelt es sich um eine Art psychologischen Automatismus, wie dies durch Humes kausale Motivationstheorie und seine (volitional gedeutete) These von der Vernunft als „Sklavin der Leidenschaften“ nahegelegt wird? Und was genau ist das Produkt von praktischen Überlegungen dieses Typs? Ist es die zweckgerichtete Handlung selbst, der Wille, sie auszuführen, oder zunächst nur der Wille, den Willen zur Ausführung auszubilden? Je nachdem, wie diese Fragen beantwortet werden, ändert sich auch die Antwort auf die Frage der volitionalen Ergebnisoffenheit.

In einfachen Fällen kann das Modell des psychologischen Automatismus ausreichen, auch in der Form des direkten Eintritts ins Handeln. Man denke etwa an Situationen wie das Aufdrehen eines Wasserhahns, um Wasser fließen zu lassen, Ausweichmanöver beim Autofahren oder das Sich-Kratzen am Kopf, um einen Juckreiz abzustellen. Allerdings liegen solche Beispiele nah bei Fällen, in denen man zweifeln kann, ob wir es überhaupt mit zweckrationalem Handeln zu tun haben und nicht nur mit einem habituierten Reflex. Auch die Ausbildung des Willens zum Ergreifen eines bestimmten Mittels kann manchmal automatisch erfolgen, z.B. beim Suchen nach einem Stift zum Schreiben oder beim Ändern der eigenen Gehrichtung, um einem anderen auszuweichen. Anhänger Humes oder anderer Empiristen tendieren dazu, auch komplexere Willensbildungsprozesse nach diesem Muster zu deuten. Manche Entscheidungstheoretiker gehen sogar soweit, überlegungsabhängige soziale Koordinationsprozesse durch Computerprogramme zu simulieren und das staunende Publikum mit bunten Grafiken von ihrem Verlauf zu erfreuen. Wie weit das möglich ist und der menschlichen Realität entspricht, mag hier dahingestellt bleiben. Klar ist jedenfalls, daß das Schema des psychologischen Automatismus dort, wo die willensbildenden Überlegungen etwas komplexer und ausgedehnter sind, meist nicht zu unserer Erfahrung paßt.

Besonders klar ist das beim Übergang direkt zum Handeln. Bei Aristoteles etwa werden als Beispiele für praktisches Schließen auch Gedankengänge wie diese angeführt: "Ich will ein gesunder Mensch sein. - Spazierengehen ist gesund. - Schon gehe ich." ${ }^{2}$ Das ist natürlich grotesk. Kein vernünftiger Mensch wird so schließen. Allenfalls kann sich der spontane Wunsch oder Wille nach einem Spaziergang einstellen. A ber auch das ist nicht der Normalfall. Das allermindeste, was noch hinzukommen muß, ist die (empi-

Vgl. Hume, Treatise II, 3,1-3; Enquiry Concerning Human Understanding, sect. VII-VIII.

Adaptiert und kontrahiert aus De Motu Animalium 701a6-70lbl; vgl. auch Ethica Nicomachea $1147 \mathrm{a}-1147 \mathrm{~b} 2$. Im Text (701a13f.) wird sogar vom Gehenmüssen jedes Menschen direkt zum eigenen Gehen übergegangen. Daß man aus solchen Passagen allerdings nicht ableiten kann, daß Aristoteles nur solche simplifizierten Schlußformen im Auge hatte, zeigt A. Kenny, Aristotle's Theory of the Will, New Haven 1979, 142f. 
risch ebenfalls seltene) subjektive Gewißheit, nur dieses eine Mittel zu haben. Mit dieser Voraussetzung war Kant bereit, das Wollen des Mittels als analytisches Implikat des gewollten Zwecks aufzufassen, jedenfalls bei vernünftigen Menschen.' Doch das geht nur mit Hilfe eines Vernunftbegriffs, der immer noch stark verkürzt.

Normale Uberlegungen verlaufen doch vielmehr so: Wenn wir erkennen, daß ein gewollter Sachverhalt ,p" nur zusammen mit "q" zu verwirklichen ist, haben wir zwar das Bewußtsein, daß wir (gemessen an den Regeln für eine realistische Willensbildung)

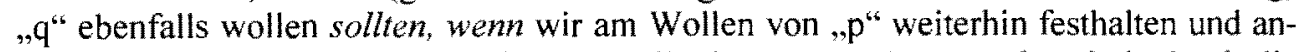
dere Optionen nicht gegeben sind. Aber weil wir uns zunächst unsicher sind, ob wir die Optionenermittlung schon weit genug vorangetrieben haben, und weil wir uns erst einmal klar darüber werden müssen, wie wir zu , $\mathrm{p}^{\text {*k }}$ und mit ihm eventuell konkurrierenden anderen Willensinhalten stehen, werden wir vernünftigerweise nicht sogleich zum WolJen von "q" (oder gar dessen Realisierung) übergehen, sondern weitere Überlegungen anstellen. Erst nachdem wir abschließend Stellung bezogen haben, ergibt sich daraus ein rationales, optional spezifiziertes Wollen und (eventuelles) Handeln. Und selbst dann ist die Umsetzung des Ergebnisses ein Schritt, der nicht immer vollzogen wird. Auch wenn wir als ,volitiv Orientierte" keinerlei Zweifel mehr haben, daß wir mit dem Rauchen aufhören oder bittere Medizin schlucken sollten, heißt das eben nicht unbedingt, daß wir den Willen dazu auch haben, geschweige denn danach handeln. Und selbst hier erscheint die pauschale Rede von "praktischer Irrationalität" unangebracht oder weltfremd. Denkbar ist allenfalls, daß wir als rationale Wesen nicht umhin können, den Willen, diese Mittel zu wollen, auszubilden und dann vielleicht nach Wegen zu suchen, diesem Anspruch gerecht zu werden.

\section{Signifikanz höherstufigen Wollens}

Könnte diese letztere Möglichkeit aber nicht ausreichen, um den prinzipiell nicht ergebnisoffenen Charakter praktischer Überlegungen zu retten? Beschränkt auf die optionale Willensbildung könnte man etwa folgendermaßen argumentieren. Präferentielles Gleichgewicht zwischen gewollten Optionen ist zwar nicht auszuschließen, läßt sich aber durch Einsatz von Zufallsgeneratoren als Mittel zu übergeordneten Zwecken auflösen. Alle Mittel gehören zu den Optionen und müssen wie diese gewollt werden. Unter gewissen Umständen geschieht das automatisch. In komplexeren Fällen treten wir in regelgeleitete Überlegungen ein, die volitional zunächst ergebnisoffen sind. Sie enden jedoch, vernünftig durchgeführt, immer in einem Wollen - zwar keinem konkreten, handlungsbezogenen Wollen erster Stufe, wohl aber in einem entsprechenden Wollen zweiter. Danach hören die Überlegungen auf. Wenn jetzt noch etwas offen ist, seien es Fragen der Handlungsmotivation oder der weiteren Willensentwicklung, kann die Ent-

Vgl, Grundlegung zur Metaphysik der Sitten, AA IV, 417. Der Text ist mehrdeutig. Teilweise klingt es so, als wolle Kant das Wollen des Mittels einfach aus einer entsprechenden Definition des "Zweckwollens" ableiten. Ich ignoriere diese Variante, da sie das Sachproblem zu einer bloßen Wortfrage machen würde. 
scheidung nicht mehr überlegt fallen, sondern nur in Abhängigkeit von anderen Faktoren. Folglich sind willensbildende Überlegungen prinzipiell nicht ergebnisoffen.

Dieses Argument hat mehrere Schwachstellen. Wird zwischen gleichgewichtigen Optionen nur per Zufall entschieden? Und vor allem: Zwingt die Einbeziehung der Folgen und Nebenfolgen nicht auch dazu, Zwecke zu problematisieren, einschließlich der bislang unbestrittenen und höchstpräferenzierten? Wenn der Rekurs auf das höherstufige Wollen etwas zugunsten der Nichtergebnisoffenheit ausrichten kann, muß es sich auch und vor allem bei der Zwecksetzung bewähren. Wie also steht es damit?

Höherstufiges Wollen und Wünschen hat in der Philosophie lange keine besondere Rolle gespielt und wurde erst durch Frankfurt wieder ins Blickfeld gerückt. Die Möglichkeit einer optativischen Stellungnahme nicht nur zu dem, was andere wollen, sondern auch zu den eigenen Wünschen und Willensinhalten ist aber seit langem bekannt. Zahlreiche positive Belege finden sich nicht nur in religiösen Texten und in der Dichtung, 'sondern auch in der Philosophie, ansatzweise z.B. bei Platon und Thomas von Aquin, deutlich bei Augustin. ${ }^{2}$ Auch der Zusammenhang mit dem Willensfreiheitsproblem ist von Augustin und anderen Philosophen (meist in kritischer Absicht) klar herausgestellt worden. Neu bei Frankfurt ist seine eigene, positive Version und die definitorische Verknüpfung mit dem Begriff der „Person". ${ }^{3}$ Beide Aspekte kann ich hier nur streifen. Mir geht es speziell um die Rolle des höherstufigen Wollens in willensbildenden Überlegungen.

Philosophen wie Hobbes, Locke, Edwards, Herbart, Schopenhauer, Ryle und viele andere haben geltend gemacht, daß die Rede von einem "Wollen des Wollens" unsinnig sei, da sie in einen Regreß führe. ${ }^{4}$ Tatsächlich kann ein Regreß entstehen, freilich nur

Im Rahmen der jüdisch-christlichen Tradition ist dies z.B. überall dort der Fall, wo ausdrücklich von der willentlichen Selbstunterwerfung des Menschen unter den Willen Gottes die Rede ist, implizit auch im Gebot der universalen Gottesliebe nach Exodus 6/5, 10/12 und Matthäus 22/37 parr. Ein schönes Beispiel für den reflektierten Selbstunterwerfungsakt findet sich etwa in J.S. Bachs Kantate Nr. 163 (Text Salomon Franck, 1715), wo der Gläubige Gott darum bittet, ihm seinen eigenen Willen zu nehmen. Erschreckende Belege fur die Übertragung dieses Gedankens auch auf die totale Unterwerfung unter kirchliche Autoritäten vermittelt W. James, The Varieties of Religious Experience, ${ }^{2} 1902$, repr. New York $1963,310-315$.

Hinweise auf reflektiertes, höherstufiges Wollen sind aber nicht auf den religiösen Kontext beschränkt, sondern finden sich vielfach auch in der weltlichen Dichtung. Ein markantes Beispiel ist die emphatische Äußerung des Ippolit in Dostojewskis Roman Der Idiot (II,10): "Jetzt will ich nichts mehr, ich will auch nichts mehr wollen, ich habe mir das Wort gegeben nichts mehr zu wollen." Ein anderes Beispiel liefert G.B. Guarinis Schäferspiel II Pastor Fido (1595, vgl. III,6), in dem der verliebte Mirtillo schwört, er könne mit keiner anderen als Amarillis glücklich sein, selbst wenn er es wollte, und er werde, sollte sein Wille es dennoch wollen, zum Himmel und seiner Liebe beten, sie möchten ihm jedes Wollen und jedes Können nehmen.

2 Vgl. Platon, Charmides 167E; Thomas v. Aquin, Summa Theologica 1II, q.I a.I ad 2, q.6 a.4 resp.; Augustin, De Libero Arbitrio 1, 12,26, 13,29; II, 19,51 1I1, 3,7-8; ders. Confessiones VIII, 5,10, 8,20-24; ders., De Civitate Dei V, 9-10; ders., De Trinitate X, 11,18; ders., Retractationes I, 13,5.

3 Vgl. insbesondere Frankfurt 1988, 19ff.

$4 \mathrm{Vgl}$. Th. Hobbes, Elements of Law I, 12,5; J. Locke, Essay Il, 21, 23, 25; J. Edwards, Freedom of the Will II, 1-2, 4-5, 7. J.F. Herbart, Lehrbuch zur Einleitung in die Philosophie \$128; A. Schopen- 
dann, wenn zugleich der Anspruch erhoben wird, das Willensfreiheitsproblem damit komplett zu lösen.' Das Phänomen selbst kann man schwerlich bestreiten. Und natürlich hat es auch Sinn, auf der (sagen wir) zweiten Reflexionsstufe zu fragen, ob man frei ist, den Willen zu haben, den man haben will. Fraglich kann nur sein, an welchen Stellen optativische Einstellungen zweiter oder höherer Stufe auftreten und welche Rolle sie in praktischen Überlegungen spielen.

Um diese Fragen beantworten zu können, muß man zwei wichtige Unterscheidungen treffen. Erstens stellt sich die Frage, worauf sich das höherstufige Wünschen und Wollen richtet: Geht es tatsächlich um die Existenz des nächstniedrigeren Wünschens und Wollens? Oder geht es lediglich darum (was bei Frankfurt im Zentrum steht ${ }^{2}$ ) die Motivationskraft und eventuelle Handlungswirksamkeit einer optativischen Einstellung, die bereits vorliegt, zu ändern oder zu bekräftigen? Zweitens sollte man positive und negative Varianten trennen: Geht es darum, daß eine bestimmte Einstellung erst ausgebildet bzw. motivational verstärkt wird? Oder soll eine bestehende Einstellung abgeschafft bzw. motivational abgeschwächt werden, so daß sie z.B. nicht mehr zum Handeln führt? Je nachdem, welchen Fall man ins Auge faßt, ändert sich auch die Signifikanz der Höherstufigkeit.

Eine umfassende Bestandsaufnahme ist hier nicht möglich. Ich beschränke mich auf Grundsätzliches. Optativische Stellungnahmen zur Motivationalität schon bestehender Wünsche und Willensregungen sind keine Seltenheit. Sie treten so gut wie immer auf, wenn wir uns reflexiv fragen, was wir als nächstes oder langfristig tun sollen. Nachfolgende Handlungsentschlüsse sind von ihnen getragen. In vielen Fällen folgt die gewünschte Motivationsänderung oder -bekräftigung problemlos und in der Form eines „psychologischen Automatismus". In manchen Fällen folgt sie dagegen nicht. Wir alle erfahren das leidvoll, wenn uns ungewollt eine boshafte Bemerkung entschlüpft, wenn wir ein Laster nicht abstellen oder uns nicht dazu aufraffen können, etwas Gewolltes und reflexiv hoch Präferenziertes konsequent umzusetzen. Aber auch in diesen Fällen, scheint mir, ist die Überlegung mit der Ausbildung der höherstufigen Einstellungen (die sich freilich nicht nur auf das Ziel, sondern auch auf den Einsatz geeigneter, selbstma-

hauer, Preisschrift über die Freiheit des Willens 1,1: G. Ryle, The Concept of Mind, London 1949, ch. III, 2 .

I Vl. dazu Seebal 1993, a.a.O., 29 A., 28, 31 A., 38, 52 A., 77, und ders., When is an Action Free?, in: G. Holmström-Hintikka / R. Tuomela (eds.), Contemporary Action Theory, vol. 1, Dordrecht $1997,237-239$.

2 Das gilt ungeachtet der Tatsache, daß Frankfurt auch Formulierungen verwendet (, A wants to want to $X^{\prime \prime}$, ,he wants to have a desire to $X^{"}$ ), die eine existenzbezogene Auffassung nahelegen (vgl. etwa 1988, a.a.O., 12f., 14, 16, 19, 22). Denn beide Formen des Wünschens zweiter Stufe, die Frankfurt in Rechnung stellt, nämlich ,second order desires" und „second order volitions", sind, wie die Definitionen und die Beispiele zeigen (1988, 13ff.), motivational spezifiziert, und entsprechendes gilt für den Begriff der Willensfreiheit (vgl. 1988, 20, 25). Auch der Willensbegriff selbst wird von Frankfurt ausdrücklich handlungsmotivational definiert (1988, 14, 164). Das entspricht, wie ich andemorts detailliert zu zeigen versucht habe (Seebaß 1993, a.a.O., Kap. I1I,3, Kap. IV), weder dem gewöhnlichen Sprachgebrauch noch einer sachlich angemessenen Konzeptualisierung und dürfte der Hauptgrund sein, warum Frankfurts Differenzierung des höherstufigen Wollens und Wünschens seltsam eklektisch und unsystematisch wirkt. 
nipulativer Mittel richten) beendet. Ob es dann zum Erfolg kommt oder nicht, ist Sache anderer Faktoren. Unsere Freiheit ist eben, auch mit Bezug auf die eigene Motivationalität, eingeschränkt.

Ähnlich verhält es sich bei der Negativariante der existenzbezogenen höherstufigen Einstellungen. Wünsche und Willensregungen, die uns hartnäckig verfolgen, können wir zumeist nicht einfach durch unseren reflektierten, gegenteiligen Willen abschaffen oder auch nur aus dem Bewußtsein verdrängen. Allenfalls können wir uns (freilich mit durchaus offenem Erfolg) für den Einsatz selbstmanipulativer Mittel entscheiden. Glücklicherweise sind Negativfâlle wie diese im Alltag relativ selten. Wenn sie auftreten, erlangen sie allerdings - unglücklicherweise - oft ein sehr hohes Gewicht.

Die korrespondierende Positivvariante ist ebenso hochgewichtig und selten. Doch liegt das nun, anders als bei den Negativfällen, keineswegs daran, daß wir so selten Gelegenheit hätten, Wünsche und Willensregungen, die wir noch nicht haben, auszubilden. Ganz im Gegenteil. In diese Lage kommen wir ständig, nicht nur bei der optionalen Willensbildung, sondern auch bei der Zwecksetzung. Nur spielen höherstufige Einstellungen dabei eine weit geringere Rolle. Normalerweise genügt es, mögliche Gegenstände des Wollens oder Wünschens zu erfassen, um direkt optativisch zu ihnen Stellung zu nehmen oder indifferent zu bleiben. Nur wenn Zweifel entstehen, etwa weil der betreffende Gegenstand sogleich als unvereinbar mit anderen (faktischen oder möglichen) Willensinhalten erkannt wird, kann der Schritt in die nächsthöhere Reflexionsstufe naheliegen, muß es aber auch nicht in jedem Fall. Wenn er erfolgt, entspricht die Situation, was die weitere Willensbildung betrifft, wieder der Negativvariante. Doch das ist hier eben nur eine Möglichkeit und zudem eine relativ seltene.

Der Rekurs aufs höherstufige Wollen ist also kein universelles Hilfsmittel bei der Analyse der Willensbildung. Auch bei der Zwecksetzung und der Wahl zwischen möglichen Zwecken haben wir mit Fällen der direkten, nicht reflexiv vermittelten optativischen Stellungnahme zu rechnen, wie sie ähnlich auch bei der Wahl zwischen gleichgewichtigen Mitteln vorkommt. Außerdem stellt sich natüllich die Frage, wie es zum höherstufigen Wollen und Wünschen kommt, zum existenzbezogenen ebenso wie zum rein motivationalen. Spielen in all diesen Fällen Überlegungen keinerlei Rolle, so daß wir durchweg (und nicht nur manchmal) bloße Beobachter dessen sind, was sich volitional mit uns vollzieht? Ich denke nicht, und ziehe daraus den Schluß, daß die These vom prinzipiell nicht ergebnisoffenen praktischen Überlegen unhaltbar ist. Wenn wir zu überlegen beginnen, ist volitiv zunächst vieles fraglich. Antworten ergeben sich teils aus der theoretischen Optionenermittlung und der Bewußtmachung schon bestehender optativischer Ansprüche, teilweise auch einfach daraus, daß etwas ohne Überlegung mit uns geschieht. Das aber erschöpft die Alternativen nicht. Offenbar gibt es Formen des Uberlegens, die auch in volitionaler Hinsicht ergebnisoffen sind - glücklicherweise. Denn da alle faktischen Willensinhalte, einschließlich der höchstrangigen, prinzipiell problematisierbar sind (vgl. 199f), blieben wir sonst im Wollen prinzipiell desorientiert. 


\section{Entscheidung ergebnisoffener Überlegungen}

Der konkrete Verlauf ergebnisoffener Überlegungen ist variantenreich und kann hier nicht im Detail untersucht werden. Ich beschränke mich auf einige Bemerkungen über ihre Entscheidung. „Subjektivistische“ und „objektivistische" Antworten sind denkbar. Letztere orientieren sich einmal mehr am Vorbild des theoretischen Wissens. So wie es objektive, erkennbare Wahrheiten gibt, so soll es auch objektive Forderungen geben, die optativische Einstellungen ebenso rational unausweichlich machen wie erkannte Wahrheiten assertorische. Als Beispiele ließen sich Kants Lehre vom kategorischen Imperativ als „Faktum der Vernunft“ anführen oder Spielarten des Werterealismus. Objektivistische Theorien aber müssen wenigstens zwei extrem starke Prämissen machen: Sie müssen zeigen, daß es die objektiven Forderungen tatsächlich gibt und daß ein rationaler Wille tatsächlich nicht umhin kann, ihnen zu folgen. Die erste Prämisse ist notorisch fragwürdig und auch die zweite ziemlich dubios. Denn warum sollte es unvernünftig (und nicht nur dissident oder unmoralisch) sein, das objektiv Geforderte nicht zu wollen? Kant hat dies mit seinem emphatischen, moralisch spezifizierten Begriff der „praktischen Vernunft“ auszuschließen versucht. Aber besitzen Menschen eine solche Vernunft wirklich?

Ich zweifle daran und werde mich deshalb bis auf weiteres nur an ,, subjektivistische“ Theorien halten, die Forderungen allein auf die optativischen Ansprüche von Personen zurückführen. ${ }^{1}$ Wie können Menschen sich orientieren, wenn sie vor der (prinzipiell ergebnisoffenen) Frage stehen, wie sie sich zu einem oder mehreren möglichen Willensinhalten stellen? Dabei mag jetzt unentschieden bleiben, ob es um ein Wollen höherer Stufe geht oder nicht, um Lebensziele oder begrenztere Zwecke oder einfach um die Entscheidung zwischen zwei gleichgewichtigen Mitteln. Drei Orientierungsformen möchte ich unterscheiden.

Die erste ist die bewußte, aktive Dezision. Man selbst nimmt so oder anders Stellung zur Sache und damit ist sie erledigt - einstweilen jedenfalls, spätere Revisionen nicht ausgeschlossen. Die zweite Form ist die Fundierung der optativischen Stellungnahme in anderen Haltungen, insbesondere eigenen Wertungen und Gefühlen. Holmer Steinfath hat diese Möglichkeit eingehend analysiert und plausibel zu machen versucht. ${ }^{2}$ Die dritte Form schließlich, die Harry Frankfurt vor allem in seinen späteren Arbeiten entwikkelt hat," ist eine Art ",volitionaler Selbsterfahrung “. Sie ist kein einmaliger Akt, son-

' Das schließt nicht nur menschliche Individuen ein, sondern auch Kollektive (,juristische Personen“) und göttliche Personen, die als Träger religiöser Sollensansprüche gelten. Auch allgemeingültige Forderungen sind im Rahmen subjektivistischer Theorien denkbar, dann nämlich, wenn es optativische Ansprüche gibt, die de facto von allen Personen erhoben werden, was keineswegs nur (sozial, kulturell oder historisch) relativistisch gedeutet werden kann, sondern auch universalistisch. Ebensowenig muß das Faktum der optativischen Allgemeinheit immer ein kontingentes sein, sondern kann sehr wohl tiefere (anthropologische) Gründe haben.

2 Steinfath 1999, a.a.O., Kap. 5-6.

3 Anlaß dafür war Frankfurts Skepsis gegenüber der volitionalen Orientierung mit Hilfe von Dezisionen. Weil es nicht möglich sei, Situationen der Desorientiertheit oder des hochrangigen präferentiellen Gleichgewichts tragfähig (,,reliably“) zu überwinden „,by making a decision“" $(1988,85)$, 
dern ein zeitlich länger erstrecktes, quasi-experimentelles „Austesten“ dessen, welche der (vorgegebenen oder durch momentane Entscheidung begründeten) optativischen Ansprüche sich dauerhaft als ,,befriedigend" oder ,,persönlich tragfähig" erweisen und dadurch (im Sinne Kants, 191) ,unerschütterlich orientierend" werden. Vor allem im Blick auf langfristige, hochrangige Ziele wie berufliche Eignung, Partnerschaft oder Weltanschauung gewinnt diese Form ihre Plausibilität. Mit ihr kommt - wie auch beim Rekurs auf subjektive Wertungen und Gefuhle - ein passivisches Moment ins Spiel, das der reinen Dezision fehlt und die praktische Orientierung der theoretischen Selbstvergewisserung annähert, ohne doch mit ihr zusammenzufallen. ${ }^{1}$

Alle drei Formen schließen einander nicht aus. Sie können und müssen sich wechselseitig ergänzen, zumindest in Fällen der Lebensorientierung. Aber der Anteil der einzelnen Formen kann unterschiedlich sein. Frankfurt und Steinfath haben sich - darin völlig im Einklang mit einer breiten Strömung der Philosophie des 20. Jahrhunderts - der Dezision gegenüber skeptisch gezeigt und ihre Bedeutung stark heruntergespielt. ${ }^{2}$ Deshalb erscheint es mir angebracht, hier eine Lanze für sie zu brechen.

bleibe als Orientierungspunkt für den Betroffenen letztlich nur das im Lebensvollzug zu ermittelnde ,fact that it is possible for him to care about the one and not about the other, or to care about the one in a way which is more important to him than the way in which it is possible for him to care about the other" $(1988,94)$. Nicht durch Akte der Dezision, sondern aus dem lebenserfahrenen, wenngleich reflektierten und wertenden Verständnis der eigenen ,psychischen Verfaßtheit" (,,of how things are with him“, vgl. 1999, 105) ergebe sich eine Haltung faktischer "Befriedigung" (,,satisfaction") über die eigene Willenslage, ohne Streben nach Änderung (1999, 102ff., vgl. auch 1988, 168f.). Als Resultat des Prozesses „volitionaler Selbsterfahrung“ ist Frankfurts Rede von „volitionaler Notwendigkeit“ (197f. Anm. 3, 4) besser verständlich, bleibt aber unvereinbar mit dem Gedanken der Autonomie, solange das passivische Moment der "Erfahrung" nicht durch die Aktivität bewuBter Dezisionen ergänzt wird (vgl. Anm.1, 2 auf 208, Anm. 1. 210).

1 Sie würden mit ihr zusammenfallen, wenn subjektive Wertungen und Gefühle bzw. die Faktoren, die für die Ergebnisse der Selbsterfahrung entscheidend sind, durchweg als vorgegebene und fixierte aufgefaßt werden könnten. Doch das ist nicht bzw. nur partiell der Fall. Sie können sich, nicht anders als optativische Stellungnahmen, mit dem Fortgang des Lebens ändern und werden dabei stets auch von Dezisionen mitbestimmt. Das passivische Moment beruht teils auf den (genetischen, traditionalen, individuell-sozialisatorischen oder durch eigene Lebenserfahrung entstandenen) Vorgaben, teils aber auch auf Kontingenzen der jeweiligen Lebenssituation. Auch diese letzteren sind für den ergebnisoffenen Charakter spezifisch praktischer Überlegungen verantwortlich. Aber sie sind es eben durchaus nicht allein, sondern werden ergänzt durch aktive, nichtkontingente Dezisionen (vgl. auch 209f.).

2 Vgl. insbesondere Steinfath 1999, Kap. 3.1 und 3.6.1, Kap. 9.3, Kap. 10.3; Frankfurt 1988, 84f. 167ff.; 1999, 100t. 112. Trotz ihrer Skepsis haben beide Autoren die Relevanz von Dezisionen anerkannt und ihren (wenn auch begrenzten) Anteil an der Willensbildung aufrechterhalten wollen. Das führt vor allem bei Frankfurt immer wieder zu einem verwirrenden Changieren zwischen bejahenden und verneinenden Aussagen (vgl. 197 Anm. 4, 208 Anm. 1). Das aktivische Moment möchte er festhalten (vgl. z.B. 1988, 87f,, 92-94, 170f:; 1999, 116). Unangemessen erscheinen ihm Dezisionen vor allem im Hinblick auf ihre vermeintliche Arbitrarität und völlige Abgelöstheit von persönlichen Vorgaben, die dazu führen müsse, daß momentane dezisionistische Akte sich nicht als dauerhaft tragfahig erweisen und ihre lebensorientierende Funktion einbüßen (vgl. bes. 1988 , 167ff.). 
Auch Erfahrungen des „befriedigten Einsseins mit uns“ oder Gefühls- und Wertungserlebnissen gegenüber gibt es nicht nur die Möglichkeit der reaktiven, passivischen Entwicklung von optativischen Einstellungen, die sie zum Inhalt haben. Es gibt auch die Möglichkeit, sie selbst - als mögliche Gegenstände der optativischen Stellungnahme - zu problematisieren und erst danach aktiv Stellung zu nehmen. Ja, diese Form der aktiv-optativischen Selbstaneignung, die auch Frankfurt bezeichnenderweise in der aktivischen Wendung des ,entschiedenen Sich-Identifizierens mit einem Wollen" beschrieben hat, ${ }^{1}$ erscheint mir unerläßlich, um jenem Gedanken der Autonomie genüge zu tun, der für unser Selbstsein konstitutiv ist (vgl. 197f. Anm.4). Wo immer sie fehlt, leben wir nicht, sondern werden gelebt - im personalen Sinne natürlich, nicht im rein biologischen. In dieser Hinsicht unterscheiden sich auch die beiden nichtdezisionistischen Orientierungsformen partiell (207, Anm. 1) nicht von der unreflektierten Übernahme kultureller oder individuell-sozialisatorischer Vorgaben (298f.) oder der Entscheidung praktischer Fragen durch Zufall und andere Determinanten. ${ }^{2}$

Die Einwände der Arbitrarität und Abgelöstheit halte ich nicht für berechtigt (siehe unten 209f.). Auch glaube ich nicht, daß Frankfurt selbst das aktivische Moment ohne Rekurs auf bewußte Dezisionen aufrechterhalten kann (vgl. 207, Anm. 1). Daß einzelne dezisionistische Akte die Dauerhaftigkeit ihres Resultats nicht garantieren können, trifft zu, zeigt aber nur, daß unser Leben auch von Vorgaben und diversen anderen Faktoren abhängig ist und daß alle optativischen Stellungnahmen prinzipiell problematisierbar und revidierbar bleiben. Wie tragfähig sie auf Dauer sind, zeigt sich erst in der "volitionalen Selbsterfahrung". Diese behält ihr Recht, stellt dasjenige von Dezisionen aber nicht in Frage, sondern kann und muß durch sie komplettiert werden.

1 Frankfurt 1988, 21f. An der aktivischen Rede vom entschiedenen "Sich-Identifizieren“ (oder sogar „Sich-selbst-Konstituieren“ durch diesen Schritt) hat Frankfurt auch festgehalten, nachdem er dezisionistische Deutungen ausdrücklich zurückgewiesen und durch sein Konzept der "volitionalen Selbsterfahrung" ersetzt hatte (vgl. etwa 1988, 170ff.; 1999, 105f.). An die Stelle der bewußten Dezision soll nun allerdings ein Begriff der „Identifikation“ $(1999,105)$ bzw. des ,decisive commitment" (1988, 168f.) treten, der letztlich nicht mehr beinhaltet als die faktische, wenngleich volitiv reflektierte, Abstandnahme eines ,,befriedigten“ (206f, Anm. 3) Individuums von weiteren Überlegungen in dem Interesse, seine volitionale Situation eventuell zu verändern.

Damit aber geht das aktivische Moment verloren bzw. überlebt allenfalls verbal. Denn da das bloße Faktum der volitionalen Höherstufigkeit, wie Frankfurt einräumt (1988, 166f.), in dieser Frage nichts entscheiden kann, bleibt, wenn Dezisionen konsequent ausgeklammert werden, nichts mehr übrig, was aktive Formen volitionaler "Befriedigung" von beliebigen anderen abheben könnte. Entsprechendes gilt für Verbalerklärungen, wonach die "Aktivität" einer Person z.B. in deren Handeln „aus ihrem eigenen Willen" bestehen soll $(1988,88)$, weil die Frage der relevanten „Eigenheit" dieses Wollens sogleich zum Problem ihres aktiven ,.Sich-Identifizierens" mit ihm zurückführt. Daß Frankfurt trotz dieses offenkundigen Defizits seiner expliziten Erklärungen an den aktivischen Redewendungen festhält, könnte ein Indiz dafür sein, daß er sich implizit doch weiterhin auf ihre dezisionistischen Konnotationen verläßt.

2 Auch solche Faktoren sind bei konkreten Willensbildungsprozessen so gut wie immer mitbeteiligt. Das notwendige Zusammenspiel von selbständiger, situativer Entscheidung und Rücksicht auf die jeweiligen hereditären, kulturellen und individuell-sozialisatorischen Vorgaben bei der Lebensorientierung ist klar herausgearbeitet bereits bei Cicero, De Officiis I, 107-121. 


\section{Falsche Vorbehalte gegen Dezisionen}

Vorbehalte gegenüber dem Dezisionismus entstehen vor allem, weil falsche Vorstellungen mit ihm verbunden werden. Vier von ihnen möchte ich hier deshalb ausdrücklich zurückweisen.

Erstens wird häufig geltend gemacht, mit Entscheidungen dieses Typs würde indeterministische Willensfreiheit unter Beweis gestellt. Doch das ist weit übertrieben. Richtig ist sicherlich, daß wir, wenn wir zu offenen Optionen selbstảndig Stellung nehmen, nicht das Bewußtsein haben, darin determiniert zu sein. Ich glaube auch, daß ohne diese Voraussetzung ergebnisoffene praktische Überlegungen sinnlos wären und die Idee der Täterschaft illusionär. Darin folge ich einer langen Denktradition, die von Aristoteles und Alexander von Aphrodisias über die Kirchenväter Nemesios und Johannes Damaszenus, Hobbes' Gegner Bramhall, Reid und Herbart bis hin zu Hart und Chisholm reicht. ${ }^{1}$ Doch vielleicht liegt darin eben eine Illusion. Das subjektive Bewußtsein, daß es sich anders verhält, ist für sich jedenfalls kein Beweis, daß unbewußte Determinanten die Entscheidung nicht dennoch steuern, seien sie nun neurophysiologischer oder tiefenpsychologischer Art. Natürlich müßte auch dies erst einmal unter Beweis gestellt werden. Aber daß es unmöglich ist, darauf sollte und braucht sich der Vertreter dezisionistischer Stellungnahmen nicht festlegen.

Zweitens muß er sich nicht, wie manchmal vermutet, die Idee eines metaphysischen "reinen Willens" zu eigen machen," oder gar die Idee eines Cartesianischen Ego oder eines „sich selbst setzenden" Fichteschen Ich. Derart anspruchsvolle Konzeptionen sind durch die einfache Anerkennung des Phänomens der Dezision nicht zu begründen.

Ebensowenig braucht man sich, drittens, durch den alten Vorwurf beeindrucken lassen, dezisionistische Willensakte liefen auf Willkür und Zufall hinaus. Man muß sich nur strikt an den aktivischen Charakter dieser Form der optativischen Stellungnahme halten. Wer sich für eine von zwei Optionen entscheidet, ohne ein übergeordnetes Wollen als Grund dafür angeben zu können, hat nicht den Eindruck, daß das Ergebnis ihm passiv zufällt, während er interessiert, aber unbeteiligt danebensteht. Darin unterscheiden sich diese Situationen grundsätzlich von solchen, in denen ein Zufallsgenerator zum Einsatz kommt oder uns etwas schicksalhaft widerfährt. Und selbst wenn das aus-

$1 \mathrm{Vgl}$. Aristoteles, De Interpretatione 18b29ff. (weitere relevante Stellen bei R. Sorabji, Necessity, Cause and Blame, London 1980, 227 A.1), sowie zum zweiten Punkt Physica VIII und Metaphysica XII, 4-7; Alexander v. Aphrodisias, De Fato, cap. 11-12; Nemesios, De Natura Hominis, cap. 41; Johannes Damaszenus, De Fide Orthodoxa II, cap. 25; Th. Hobbes, The English Works, ed. W. Molesworth, London 1841, repr. Aalen 1966, vol. V, 150f. 279, 293; Th. Reid, Essays on the Active Powers of the Human Mind 1,1-2; IV,1-2, 6-8; J.F. Herbart, Zur Lehre von der Freiheit des menschlichen Willens, in: ders., Schriften zur praktischen Philosophie, ed. G. Hartenstein, Bd, II, Leipzig 1851, 345f.; H.L.A. Hart, Punishment and Responsibility, Oxford 1968, 28f. A.1; R.M. Chisholm, Freedom and Action, in: K. Lehrer (ed.), Freedom and Determinism 1966, 11-44; ders., Person and Object, London 1976, ch. II.

2 So Tugendhat 1992, a.a.O., 467 in der Kritik an Frankfurt. Die Kritik trifft Frankfurt nicht (vgl. 208f., Anm. 3 und 209, Anm. 2), kann aber durch dessen schwankende Haltung zur Dezision und seine aktivische Rede vom "Sich-Identifizieren" oder "Sich-selbst-Konstituieren" (u.ä., vgl. 210 Anm. 1) immerhin nahegelegt werden. 
nahmslos zuträfe, was manchmal fraglos der Fall ist, daß wir uns nämlich für etwas entscheiden, von dem wir nachher den Eindruck haben, genausowenig zu wissen, wie wir dazu gekommen sind, wie bei Zufällen und bloßen Widerfahrnissen, würde ein fundamentaler Unterschied immer noch darin bestehen, daß wir das Resultat dieser „QuasiEntscheidung“ als unseres anerkennen, uns also „mit ihm identifizieren“. Das ist eine Variante des alten Stoischen Gedankens der bewußten Einstimmung ins Unabänderliche, die jedenfalls dann keine rein fatalistische ist, wenn sie die freie, aktiv-optativische Stellungnahme des Individuums einschließt. Zumindest auf dieser Ebene aber scheint mir die passivische Rede vom „Zufallen“ oder ähnlichem extrem kontraintuitiv zu sein, sondern nur Kants Beschreibung die Sache zu treffen: „Wenn ich sage: Ich denke, ich handele usw.; dann ist entweder das Wort lch falsch angebracht, oder ich bin frei." I Und auch die Stoisch gedeuteten „Quasi-Entscheidungen“ sind ja glücklicherweise eher ein Sonderfall, der unserer Alltagserfahrung nicht entspricht.

Viertens schließlich bedeutet die Anerkennung von Dezisionen keineswegs, daß wir vollkommen frei darin sind. Nicht nur, daß uns nur ein sehr begrenzter Teil realer Optionen persönlich zugänglich ist, ontologisch wie epistemisch gesehen. Auch zwischen Optionen, denen gegenüber wir uns, was die volitionale Lage betriff, in einer prinzipiell ergebnisoffenen Wahlsituation befinden, können wir uns oft nicht völlig frei entscheiden. Kapazitätsgrenzen des Intellekts, des Gedächtnisses und anderer psychischer Faktoren wirken sich faktisch spielraumverengend aus, ebenso bestehende Habitualisierungen und Automatisierungen (196f.). Und natürlich sind auch die Beschränkungen durch subjektive Wertungen und Gefühle und durch die Selbsterfahrung in Rechnung $\mathrm{zu}$ stellen. Vor allem längerfristige Zielsetzungen, aber auch viele Alltagsentscheidungen werden von ihnen mitgeprägt. Zum Beispiel können wir uns nicht ohne weiteres dafür entscheiden, eine Speise zu kosten, vor der uns ekelt, oder ein Spiel mitzuspielen, das uns langweilig oder moralisch verwerflich erscheint. Und diese Restriktionen bestehen sehr oft auch dann, wenn wir uns über die kulturelle oder sozialisatorische Kontingenz unserer Wertungen völlig im klaren sind. Selbstmanipulative Mittel können den Dezisionsspielraum z.T. verbreitern. Manchmal genügt auch, wie schon Thomas von Aquin festgestellt hat, ${ }^{2}$ die ihrerseits auf aktiver Entscheidung fußende Konzentration auf eine bestimmte Option oder einen bestimmten Aspekt in ihr. Aber natürlich geht das nicht überall oder unlimitiert. Doch die begrenzte Bedeutung dezisionistischer optativischer Stellungnahmen anzuerkennen, heißt nicht, sie für bedeutungslos zu erklären. Im Gegenteil, gerade durch dieses Zugeständnis tritt ihre reale Signifikanz hervor.

I I. Kant, Vorlesungen über Metaphysik, AA XXVIII/1, 269. Vergleichbare Äußerungen über den intrinsisehen Zusammenhang von Ich und Aktivität finden sich öfter bei Kant, so etwa in der $A n$ thropologie (z.B. AA V1I, 161, 397f.) sowie im Paralogismuskapitel und in der Transzendentalen Deduktion der Kritik der reinen Vernunft (z.B. B 157f.).

2 Thomas v. Aquin, Summa Theologica 1II, q.10 a.2 ad 1; q.13 a.6 resp. ad. 3; ders., Quaestiones Disputatae de Malo q.6, resp. / ad 7 / ad 15. 


\section{Schluß}

Was also heißt es, sich „im Wollen zu orientieren“? Offenbar heißt es nicht nur, sich theoretisch über die eigene Willenslage ins Bild zu setzen, Optionenermittlung zu betreiben und daraus praktische Schlüsse zu ziehen. Es heißt auch und vor allem, spezifisch praktische C̈lberlegungen anzustellen, die nicht nur epistemisch, sondern auch volitional ergebnisoffen sind und deshalb auch durch aktive optativische Stellungnahmen beendet werden. Wer ,weiß, was er will“, hat eben nicht nur partielle Gewißheit darüber gewonnen, was ist, sondern auch darüber, was seinem Anspruch nach sein soll. Und dieses praktische, vom theoretischen scharf zu unterscheidende „Wissen“ ist zugleich eines, das prinzipiell (im Gegensatz zu Kants Behauptung, 191) über unsere Erfahrung hinausführt. 